\title{
Prevalence of asthma among schoolchildren in Ahvaz, Islamic Republic of Iran
}

A.H. Shakurnia, ${ }^{1}$ S. Assar, ${ }^{2}$ M. Afra ${ }^{7}$ and M.Latifi ${ }^{3}$

$$
\begin{aligned}
& \text { معدل انتشار الربو بين أطفال المدارس في الأهواز، جمهورية إيران الإسلامية }
\end{aligned}
$$

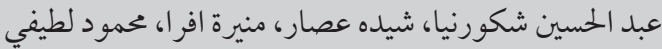

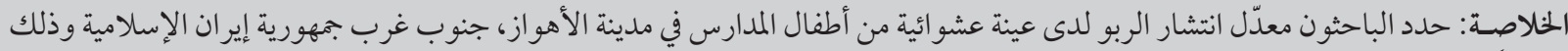

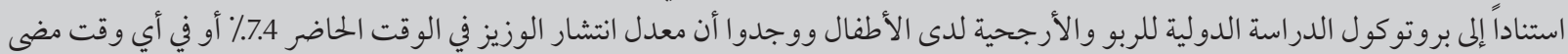

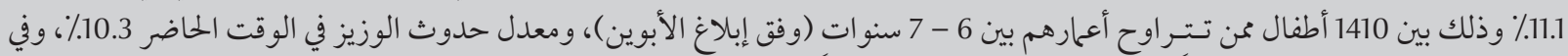

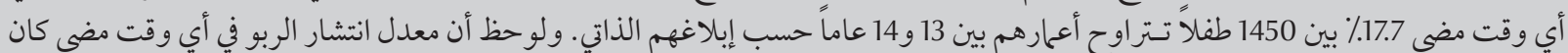

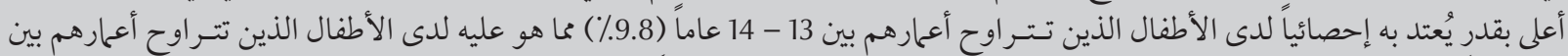

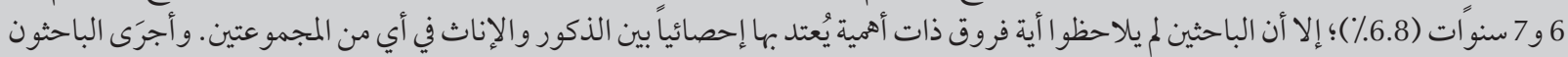

$$
\text { مقارنات مع معطيات المدن الإير انية الأخرى ومع المعطيات الدوات الدولية. }
$$

ABSTRACT The prevalence of asthma was determined in a random sample of schoolchildren in Ahvaz city, southwest Islamic Republic of Iran, based on the International Study of Asthma and Allergy in Childhood (ISAAC) protocol. The prevalence of ever wheeze and current wheeze was $11.1 \%$ and $7.4 \%$ among 1410 children aged 6-7 years (parent-reported) and $17.7 \%$ and $10.3 \%$ among 1450 children aged $13-14$ years (self-reported). The prevalence of ever asthma was significantly higher among 13-14-year-olds (9.8\%) than 6-7-year-olds (6.8\%) but no significant difference was found between males and females in either age group. Comparisons are made with other Iranian cities and international data.

\section{Prévalence de l'asthme chez les élèves d'Ahvaz (République islamique d'Iran)}

RÉSUMÉ La prévalence de l'asthme a été déterminée selon le protocole de l'Étude internationale de l'asthme et des allergies chez les enfants (ISAAC) sur un échantillon d'élèves de la ville d'Ahvaz (sud-ouest de la République islamique d'Iran) sélectionnés au hasard. La prévalence de la respiration sifflante diagnostiquée à un moment quelconque et de la respiration sifflante actuellement diagnostiquée était de 11,1\% et 7,4\% chez les 1410 enfants âgés de 6 à 7 ans (épisodes déclarés par les parents), et de 17,7\% et 10,3\% parmi les 1450 enfants âgés de 13 à 14 ans (épisodes auto-déclarés). La prévalence de l'asthme diagnostiqué à un moment quelconque était significativement plus élevée chez les 13 - 14 ans $(9,8 \%)$ que chez les 6 - 7 ans $(6,8 \%)$, mais aucune différence significative n'a été observée entre les garçons et les filles dans les deux groupes d'âge. Des comparaisons sont effectuées avec d'autres villes iraniennes et des données internationales.

${ }^{1}$ Department of Immunology; ${ }^{2}$ Department of Paediatrics; ${ }^{3}$ Department of Epidemiology and Biostatistics, Faculty of Health, Ahvaz University of Medical Sciences, Ahvaz, Islamic Republic of Iran (Correspondence to A.H. Shakurnia: shakurnia@yahoo.com).

Received: 16/03/08; accepted: 13/08/08 


\section{Introduction}

Asthma is one of the most common chronic respiratory disorders and the prevalence of asthma and atopy has been increasing worldwide over the last 2 decades, especially in children $[1,2]$. The reported prevalence of asthma and other allergic disorders varies both within and between countries [3]. International and regional comparisons of asthma have been greatly facilitated by the International Study of Asthma and Allergies in Childhood (ISAAC) programme, which has formulated a standard questionnaire to collect data on the prevalence and severity of asthma [4]. The first results of ISAAC showed that the prevalence of asthma symptoms varied in different countries from $1.6 \%$ to $36.8 \%$ [4]. Genetics, lifestyle and environmental factors may play a role in these variations [5].

Because only a limited number of studies have been conducted on asthma in the south-west of the Islamic Republic of Iran, the epidemiology of this disease is still not fully understood in the region. This study was therefore designed to determine the prevalence of asthma and wheezing among schoolchildren of Ahvaz city in Khuzestan province and to compare the results with other studies in the Islamic Republic of Iran using the same protocol. It was hoped that the study would be helpful to health system policy-makers in planning asthma prevention programmes in the region.

\section{Methods}

A descriptive cross-sectional study in 2007 was conducted on schoolchildren in 2 age groups: 6-7 and 13-14 years in the city of Ahvaz in the centre of Khuzestan province, in the south-west of the Islamic Republic of Iran. Ahvaz is in the interior at an altitude of $18 \mathrm{~m}$ above sea level. In general, the city has an extremely hot and humid climate during summer.
The population of Ahvaz is 1338000 , some of whom are of Arab descent.

\section{Sample}

The sample size was estimated as 2401 , based on an asthma prevalence of $6.7 \%$ from a study carried out in Bushehr, a city located in the south of the Islamic Republic of Iran with a similar geographic environment [6], and 95\% confidence (error 0.05 ) and accuracy $1 \%$ (d $=0.01$ ). To allow for non-responses the sample size was increased to 3000 .

The sampling frame of the survey was a list of all primary and intermediate schools provided by the Ahvaz Ministry of Education, indicating the district and region, number of schools and number of children in primary schools for the 6-7 years age group and preparatory/ intermediate for the 13-14 years age group. A total of 32 schools, 16 for the 6-7 years age group and 16 for the 13-14 years age group among the 4 region of the Ahvaz city (each region 4 schools) were chosen randomly.

Ethical approval of the study protocol was obtained from both the Ministry of Health and the Ministry of Education.

\section{Questionnaire and data collection}

We used the ISAAC questionnaire for the survey [3]. The questionnaire was translated into Farsi by a specialized medical translator. The Farsi version was revised by a consultant paediatrician and an immunologist, and then back translated into English to check its equivalence. After obtaining consent from the school authorities, the 6-7year-old schoolchildren were asked to take the questionnaires home with a letter of explanation. The questionnaire was completed by the parents of each child and returned to the school. The 13-14-year-olds completed the questionnaires in the classroom under the supervision of professionally trained interviewers according to phase 1 of the ISAAC methodology [3].

\section{Definition of terms}

The following terms were defined: ever wheeze (wheezing or whistling in the chest at any time in the past); current wheeze (wheezing or whistling in the chest at any time in the past 12 months); severe wheeze (wheezing severe enough to limit speech to only 1 or 2 words at a time between breaths); exercise-related wheeze (whistling sounds in the chest during or after exercise); nocturnal cough (dry cough at night, apart from a cough associated with a cold or a chest infection); ever asthma (ever had asthma diagnosed by physician at any time in the past).

\section{Analysis}

The questionnaires were coded, entered into the computer and processed according to ISAAC guidelines. The data were analysed using SPSS, version 15 . We calculated the prevalence for each of the symptoms and presented them as simple frequencies. The significance of difference between proportions was calculated using the chi-squared test, with $P$ value $<0.05$ as the level of significance

\section{Results}

Of the 3000 questionnaires distributed, 2860 completed questionnaires were returned, a response rate of $95.3 \%$. There were 1410 (49.3\%) children aged 6-7 years old and $1450(50.7 \%)$ aged 13-14 years old.

Table 1 shows the prevalence of wheeze and asthma in schoolchildren by age group and sex. According to parents' reports $156(11.1 \%)$ of 6-7year-old schoolchildren were reported to have ever suffered wheeze, with no significant difference between boys and girls $(11.8 \%$ versus $10.5 \%)(P=0.36)$. The questionnaire to the $13-14$ years age group showed that $250(17.7 \%)$ of them had experienced wheezing in their lifetime, $16.2 \%$ of males and $18.8 \%$ of females. Although the proportion of 


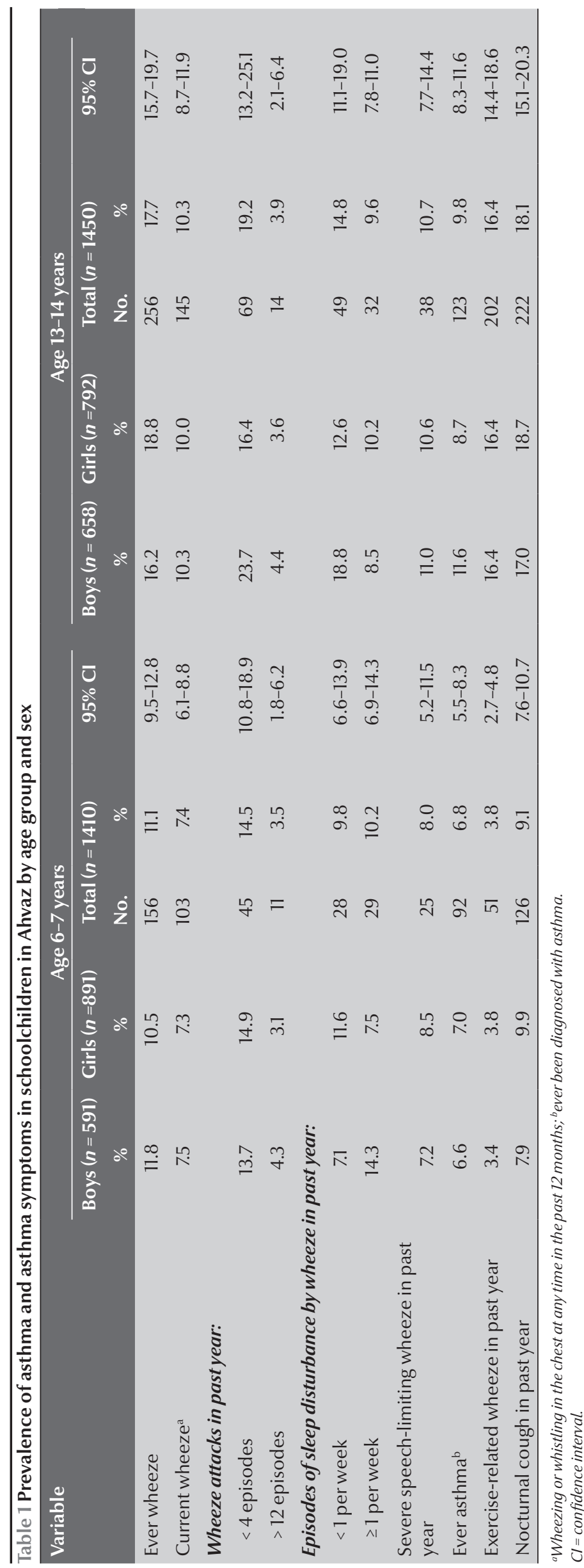

females was higher, this difference was not statistically significant $(P=0.19)$.

The prevalence of ever wheeze and the prevalence of wheeze and exercise wheeze in the previous 12 months were significantly higher in the 13-14 years age group $(17.7 \%, 10.2 \%$ and $16.4 \%$ respectively) compared with the $6-7$ years age group $(11.1 \%, 7.4 \%$ and $3.8 \%$ respectively) (Table 1 ).

Ever asthma, i.e. a history of physician-diagnosed asthma, was reported for $6.8 \%$ of the $6-7$-year-olds, $6.6 \%$ othe $f$ boys and $7.0 \%$ of the girls $(P=0.76)$ (Table 1). Ever asthma was reported by $9.8 \%$ of the 13-14-year-old schoolchildren. Although ever asthma was more prevalent in boys than girls (11.6\% versus $8.7 \%)$, the difference was not statistically significant $(P=0.10)$.

The prevalence of nocturnal cough was significantly higher in the older age group than in the younger children $(18.1 \%$ versus $9.1 \%, P<0.001)$ but was not significantly different between the sexes in either age group.

Exercise-related wheeze in the previous year was significantly higher in the 13-14-year-olds (16.4\%) than 6-7-year-olds $(3.8 \%)(P<0.01)$, but had a similar rate for boys and girls in both age groups.

No sex- or age-dominance was seen in the rate of severe asthma symptoms (sleep-disturbing and speech-limiting wheeze). A higher rate of speech limiting wheeze was reported by 13-14-year-olds compared with 6-7-year-olds, although this was not statistically significant.

\section{Discussion}

We found that the prevalence of ever asthma among schoolchildren in Ahvaz was 9.8\% in 13-14-year-olds and $6.8 \%$ in $6-7$-year-olds. Both these figures are higher than the mean prevalence of asthma in the Middle East according to data from the Global Initiative for Asthma (5.8\%) [7]. A previous study in Ahvaz in 2005 found an asthma prevalence of $8.7 \%$ [8], but the rates reported from other Iranian cities range from $2.1 \%$ to $7.1 \%$, using the ISAAC written questionnaire in 6-14year-old schoolchildren $[9,10-12]$. The prevalence of asthma in the current study using the ISAAC protocol was also lower than in neighbouring countries in the Persian Gulf region where similar surveys were done, namely Iraq, Kuwait, Oman, Qatar and Saudi Arabia [6]. The Islamic Republic of Iran ranked 28th in the 
prevalence of current asthma among the 56 countries where the ISAAC study was done.

The finding that the lifetime asthma prevalence in Ahvaz was closer to that of surroundingArab countries but different from other cities in the Islamic Republic of Iran may be explained by similarities in the climate (humid and very hot) between Ahvaz city and neighbouring Arab countries. Our results were similar to another 2 cities of Islamic Republic of Iran, Boushehr and Gorgan, both of which are located near the sea, have high humidity and low altitude, suggesting that geographical location could affect the asthma prevalence in these regions. A study in Saudi Arabia showed major differences in the prevalence of bronchial asthma comparing coastal and inland areas with different climates [13]. Other studies in Western Europe (57 centres in 12 countries) also showed an association between altitude, temperature and humidity and the prevalence of asthma symptoms in these age groups [14].

For the 6-7 years age group, the prevalence of ever asthma of $6.8 \%$ puts Ahvaz in the high prevalence category when compared with some other cities in Islamic Republic of Iran using the same ISAAC protocol (Table 2) Qazvin 2.0\% [15], Tehran 2.2\% [10] and Rasht $7.1 \%[11]$ _ and in the low prevalence category of ever asthma among countries around the Persian Gulf-Iraq 22.3\% [16], Qatar 19.8\% [17] and Oman 10.5\% [18].

Consistent with most studies in other countries, the rate of ever asthma in the 13-14-year-old schoolchildren was higher than in the 6-7-year-old schoolchildren. The prevalence of ever asthma in the 13-14 years age group in Ahvaz (9.8\%) was the highest among the 6 cities in the Islamic Republic of Iran using the ISAAC questionnaire (Table 2)_- Urmia 2.1\% [11], Qazvin $2.2 \%$ [15], Tehran 2.6\% [10], Rasht 4.7\% [11], Bushehr 6.7\% [9] and Gorgan $7.0 \%$ [19]. However, the prevalence of ever asthma in the 13-14 year age group was similarly lower than reported in this age group in neighbouring Arab countries - in Saudi Arabia 23.0\% [20], Iraq 22.3\% [16], Oman 20.7\% [18], Qa$\operatorname{tar} 19.8 \%$ [17], Kuwait 16.8\% [21] and the United Arab Emirates 13.0\% [22].

Ever wheeze showed a higher prevalence compared with ever asthma regardless of age. This might be due to wheezing resulting from causes other than asthma, but the most likely explanation is an underdiagnosis of asthma or the reluctance of physicians to label a child as asthmatic.

Evidence from the ISAAC study showed that the distribution of childhood asthma varied between global populations from less than $2 \%$ to $33 \%$ [4]. This variability may be related to differences in racial composition, environmental factors, climate, health facilities, diet and lifestyle. Since the Islamic Republic of Iran is a large country with diverse geographical areas, the different prevalences found in different cities may be due to the type of weather, the level of air pollution and genetic factors.

One striking finding in our study was that the prevalence of ever asthma and severe asthma symptoms did not differ significantly between boys and girls. Many regional studies have shown a predominance among boys in the prevalence of wheeze and ever asthma, whereas other studies from Lebanon, Turkey and India found similar rates of wheeze and ever asthma for both sexes [4,23-24].

\begin{tabular}{|c|c|c|c|c|c|}
\hline Age group/ city & $\begin{array}{c}\text { Ever wheeze } \\
\%\end{array}$ & $\begin{array}{c}\text { Current wheeze }^{\mathrm{a}} \\
\%\end{array}$ & $\begin{array}{c}\text { Ever asthma }^{\mathrm{b}} \\
\%\end{array}$ & $\begin{array}{c}\text { Sleep-disturbing wheeze } \\
\%\end{array}$ & $\begin{array}{c}\text { Speech-limiting wheeze } \\
\%\end{array}$ \\
\hline \multicolumn{6}{|l|}{ 6-7years } \\
\hline Ahvaz & 11.1 & 7.3 & 6.8 & 10.2 & 8.0 \\
\hline Tehran & 15.6 & 8.6 & 2.2 & 18.4 & 1.5 \\
\hline Rasht & 22.0 & $\mathrm{n} / \mathrm{a}$ & 7.1 & 6.0 & 12.6 \\
\hline Qazvin & 10.0 & 10.2 & 2.0 & 35.7 & $\mathrm{n} / \mathrm{a}$ \\
\hline \multicolumn{6}{|l|}{ 13-14 years } \\
\hline Ahvaz & 17.7 & 10.0 & 9.8 & 9.6 & 10.7 \\
\hline Tehran & 17.3 & 10.9 & 2.6 & 5.2 & 3.0 \\
\hline Rasht & 24.0 & $\mathrm{n} / \mathrm{a}$ & 4.7 & 4.3 & 9.7 \\
\hline Qazvin & 26.0 & 17.6 & 2.2 & 36.9 & $\mathrm{n} / \mathrm{a}$ \\
\hline Urmia & 23.7 & 14.5 & 2.1 & $\mathrm{n} / \mathrm{a}$ & $\mathrm{n} / \mathrm{a}$ \\
\hline Gorgan & 30.5 & 20.1 & 7.0 & 4.2 & 11.8 \\
\hline Bushehr & 19.8 & 10.8 & 6.7 & 10.6 & 31.8 \\
\hline Birjand & 18.0 & 10.1 & $\mathrm{n} / \mathrm{a}$ & 8.9 & 4.0 \\
\hline
\end{tabular}

${ }^{a}$ Wheezing or whistling in the chest at any time in the past 72 months; ${ }^{b}$ Ever been diagnosed with asthma; includes children awakening more than once/night per week. $n / a=$ data not available. 
As our results demonstrate, the overall proportion of girls with a positive history of ever wheeze was the same as boys, but there was a significantly higher rate in 13-14-year-olds versus 6-7-year-olds, i.e. the prevalence of ever asthma increased with increasing age, as it did in other studies $[4,10]$. If wheeze is considered the main symptom of asthma, this finding is similar those of most clinical studies: that the prevalence of asthma in adolescents is higher than in younger children.

Asthma severity is assessed in the ISAAC questionnaire by asking about sleep-disturbing wheeze and speechlimiting wheeze. As shown, no sex- or age-dominance was detected regarding these 2 indices of symptom severity. A higher rate of speech limitation was reported by 13-14-year-olds compared with the 6-7-year-old group although this was not significant. For the 6-7 years age group, the Islamic Republic of Iran ranked 27 out of 38 countries in the ISAAC global ranking for the prevalence of sleep-disturbing wheeze. Among the 3 cities in the Islamic Republic of Iran with data for this age group Ahvaz had the lowest proportion of children with severe asthma symptoms (sleep-disturbing and speech-limiting wheeze), and also among the 3 Eastern Mediterranean countries with data for this age group Ahvaz had the highest proportion of children with severe asthma symptoms [16-18]. For the 13-14 years age group, the Islamic Republic of Iran ranked 23 out of 56 countries in the ISAAC global ranking for the prevalence rate of sleep-disturbing wheeze. Among the 7 cities in Islamic Republic of Iran with data for this age group Ahvaz had the lowest proportion of children with severe asthma symptoms (sleep-disturbing and speech-limiting wheeze) and also among the 3 Eastern Mediterranean countries with data for this age group Ahvaz had the highest proportion of children with severe asthma symptoms $[17,18,21]$.

It has been estimated that wheeze and asthma symptoms improve with age and that remission of asthma is about 50\% [16]. We found, however, that the prevalence of ever wheeze, current wheeze, exercise-related wheeze and nocturnal cough in the previous year was significantly lower from the reports of parents of 6-7 year olds than the self-reports of adolescents. These findings may reflect under-reporting by parents (parents' recall bias) and/ or over-reporting by adolescents or different interpretation of questions. These biases usually accompany questionnaire-based studies.

The significantly higher rate of nocturnal cough among adolescents might be due to reports of night cough resulting from other causes, such as common colds or chest infections, because adolescents may not have the experience to differentiate the cause of their cough. Exercise may cause more wheezing in adolescents than in 6-7year-old children because the frequency of participation in competitive sports and vigorous exercise activities is higher among adolescents. Exercise-induced wheeze and night cough were reported equally by both sexes in both age groups, but at a significantly higher rate among the 13-14-year-old group.

This study had some limitations, including the possibility of recall bias in questionnaires and the lack of objective laboratory measures. The prevalence of asthma was based on data from a questionnaire with questions concerning symptoms or a previous diagnosis of asthma.

\section{Conclusions}

Our results demonstrate that Ahvaz city has a relatively high prevalence of asthma in schoolchildren compared with other cities in Islamic Republic of Iran, but a low prevalence compared with neighbouring Arab countries where the ISAAC protocol was used. The prevalence of asthma was higher in 13-14-year-olds compared with 6-7year-olds, but was approximately equal in boys and girls.

\section{Acknowledgements}

This study was approved and funded by Ahvaz Jundi-Shapur University of Medical Sciences. The authors would like to thank the schoolchildren, their parents and other staff who participated in the study.

\section{References}

1. Wong GW, Chow CM. Childhood asthma epidemiology: Insight from comparative studies of rural and urban populations. Pediatric pulmonology, 2008, 43:107-16.

2. Downs $\mathrm{SH}$ et al. Continued increase in the prevalence of asthma and atopy. Archives of disease in childhood, 2001, 84:20-3.

3. Partridge MR, Alwan A. Prevention of asthma and approaches for enhanced care in the Eastern Mediterranean Region. Eastern Mediterranean health journal, 1997, 3(1):133-43.

4. Asher MI et al. International study of asthma and allergies in childhood (ISAAC): rationale and methods. European respiratory journal, 1995, 8:483-91.
5. Ece A et al. Prevalence of asthma and other allergic disorders among schoolchildren in Diyarbakir, Turkey. Turkish journal of pediatrics, 2001, 43:286-92.

6. Beasley R. Worldwide variation in prevalence of symptoms of asthma, allergic rhinoconjunctivitis, and atopic eczema: ISAAC. Lancet, 1998, 351:1225-32.

7. Masoli M et al. Global burden of asthma. Geneva, Global Initiative for Asthma, 2004.

8. Pezeshkpour Z, Ziyaei T, Taheri M, Survey of prevalence of asthma in schoolchildren in Ahvaz [MD thesis]. Department of Pae- 
diatrics, Faculty of Medicine, Ahvaz Jundi-Shapur University of Medical Sciences, Ahvaz, Islamic Republic of Iran, 2005.

9. Hatami G et al. Prevalence of asthma and asthma-related symptoms among 13-14 yr. schoolchildren in Bushehr, ISAAC. Iranian south medical journal, 2002, 5(2):167-75.

10. Reza Masjedi M et al. Prevalence and severity of asthma symptoms in children of Tehran: International Study of Asthma and Allergies in Childhood (ISAAC). Iranian journal of allergy, asthma and immunology, 2004, 3(1):25-30.

11. Abbasi Ranjbar Z. Prevalence of asthma symptoms in children. Journal of Guilan University of Medical Sciences, 2006 56(14):1-9.

12. Rahimi Rad MH, Hejazi ME, Behrouzian R. Asthma and other allergic diseases in 13-14-year-old schoolchildren in Urmia: an ISAAC study. Eastern Mediterranean health journal, 2007, 13(5):1005-16.

13. Bener A et al. Prevalence of asthma and wheeze in two different climatic areas of Saudi Arabia. Indian journal of chest diseases and allied sciences, 1993, 35(1):9-15.

14. Weiland SK et al. Climate and the prevalence of symptoms of asthma, allergic rhinitis, and atopic eczema in children. Occupational and environmental medicine, 2004, 61:609-15.

15. Zohal MA, Hasheminasab R. Prevalence of asthma among school-age children in Qazvin (2003). Journal of Qazvin University of Medical Sciences, 2006, 9(4):64-8.
16. Al-Thamiri D, Al-Kubaisy W, Ali SH. Asthma prevalence and severity among primary-school children in Baghdad. Eastern Mediterranean health journal, 2005, 11(1/2):1179-86.

17. Janahi IA et al. Prevalence of asthma among qatari schoolchildren: international study of asthma and allergies in childhood, Qatar. Pediatric pulmonology, 2006, 41(1):80-6.

18. Al-Riyami BMS et al. A relatively high prevalence and severity of asthma, allergic rhinitis and atopic eczema in schoolchildren in the Sultanate of Oman. Respirology, 2003, 8(1):69-76.

19. Bazzazi $\mathrm{H}$ et al. The prevalence of asthma and allergic disorders among school children in Gorgan. Journal of research in medical sciences, 2007, 1(12):28-33.

20. Al-Frayh AR et al. Increased prevalence of asthma in Saudi Arabia. Annals of allergy, asthma and immunology, 2001, 86(3):292-6.

21. Behbehani NA et al. Prevalence of asthma, allergic rhinitis, and eczema in 13- to 14-year-old children in Kuwait: an ISAAC study. Annals of allergy, asthma and immunology, 2000, 85:58-63.

22. Bener A et al. Prevalence of asthma among Emirates school children. European journal of epidemiology, 1994, 10:271-8.

23. Ramadan FM et al. Prevalence of asthma and asthma symptoms in children in urban Lebanon. Saudi medical journal, 1999, 20(6):453-7.

24. Paramesh H. Epidemiology of asthma in India. Indian journal of pediatrics, 2002, 69(4):309-12.

\section{Global Alliance against Chronic Respiratory Diseases (GARD)}

GARD is a voluntary alliance of national and international organizations, institutions, and agencies commited towards the common goal to reduce the global burden of respiratory diseases.

The Global Alliance is part of the global work to prevent and control chronic diseases. Because most of the chronic respiratory diseases are under-diagnosed, under-treated and the access to essential medications in many countries is poor, a global effort to improve the diagnosis and the medical care is needed.

More information on GARD activities and publications can be found on the homepage at: http://www.who.int/gard/en/ 